\title{
Evaluation of a Modified Flow-Through Method for Predictive Dissolution and In Vitro/In Vivo Correlations of Immediate Release and Extended Release Formulations
}

\author{
Hanxi Yi, ${ }^{1}$ Fan Liu, ${ }^{2}$ Guoqing Zhang, ${ }^{1}$ and Zeneng Cheng ${ }^{1}{ }^{1}$ \\ ${ }^{1}$ Division of Biopharmaceutics and Pharmacokinetics, Xiangya School of Pharmaceutical Sciences, Central South University, China \\ ${ }^{2}$ Neurology Department, The First Affiliated Xiangya Hospital, Central South University, China \\ Correspondence should be addressed to Zeneng Cheng; chengzn@csu.edu.cn
}

Received 7 March 2021; Revised 22 March 2021; Accepted 30 March 2021; Published 28 April 2021

Academic Editor: Tingting Hong

Copyright @ 2021 Hanxi Yi et al. This is an open access article distributed under the Creative Commons Attribution License, which permits unrestricted use, distribution, and reproduction in any medium, provided the original work is properly cited.

\begin{abstract}
The present study evaluated the ability of a modified flow-through method for predicting in vivo performance of immediate release (IR) and extended release (ER) formulations. In vitro dissolution of two model drugs, paracetamol IR tablets and felodipine ER tablets, was investigated under tuned conditions using the modified flow-through method and compared with the compendial quality control (QC) basket method. The in vivo absorption properties of paracetamol IR tablets and felodipine ER tablets were investigated in healthy volunteers. In vitro-in vivo correlation (IVIVC) analysis was performed based on the obtained in vitro and in vivo data. Our results demonstrated that the compendial QC method was not able to reflect in vivo actual absorption, while satisfactory discriminatory power and comparable in vitro dissolution/in vivo absorption were achieved for both paracetamol IR tablets and felodipine ER tablets by the modified flow-through method. This study indicated that the modified flow-through method is a potential tool to reflect in vivo performance of the IR and ER formulations.
\end{abstract}

\section{Introduction}

Over the past three decades, dissolution testing methodology has been introduced in many pharmacopeias, and it is widely recognized that comparison of percent in vitro dissolution and in vivo absorption can provide useful information [1, 2]. Usually, in vitro dissolution tests are operated in vesselbased systems, commonly known as the basket and paddle apparatuses to demonstrate suitable dissolution curves of the drugs. In vitro dissolution tests can act a valuable role in formulation development and can be helpful for in vivo product characterization. One of the goals of in vitro dissolution studies is to estimate the in vivo characteristics of the formulations [3-6].

In the biopharmaceutics research area, one of persisting challenges is relating percent in vitro drug dissolved with in vivo performance [7-9]. The value of compendial dissolution methods, acted as quality control (QC) tool for forecasting the drug's in vivo performance, can be significantly increased when an in vitro-in vivo correlation (IVIVC) is achieved [10]. The term IVIVC was defined by the Food and Drug Administration (FDA) as "a predictive mathematical model describing the relationship between an in vitro property of a dosage form and an in vivo response" [11]. Lack of a correlation between in vivo behavior and in vitro dissolution data may cause undesirable management of the important production parameters based on dissolution test and limit biopharmaceutical explanation of in vitro testing data [12-14].

Both the basket and paddle approaches, acted as highly prevalent methods for common drug formulations, are conducted in closed situation (finite sink), thus cannot reflect the actual circumstances of in vivo gastrointestinal system $[15,16]$. Concerning the in vivo condition, the drug dissolution manner was mainly occurred in the site that free drugs close to or adhering to the mucosal surface. Consequently, the diffusional process to the absorption site is often fast that is resulting an infinite sink, and the free drugs are immediately entered into the body circulatory system after the dissolution. Therefore, the possibility of achieving successful 
IVIVCs in this aspect is enhanced when the in vitro dissolution tests closely approach the gastrointestinal infinite sink environment [17]. For current stationary testing systems, the necessity for keeping an infinite sink requires employing a large dissolution medium volume and high agitation speed. However, Levy et al. [18] have reported that the agitation speed should be maintained relatively low in most cases for building good IVIVCs. Also, the dissolution tests often demand to operate at low agitation for detecting slight variations between products. Unfortunately, relatively low agitation with high medium volume testing conditions causes unsatisfied homogeneity, and the withdrawn sample would not reflect the actual entire dissolved system. Therefore, an intrinsic disagreement remains in static apparatuses between the necessities for large medium volumes with low agitation speeds and homogeneity [17].

In the past, limited success has been achieved in the field of IVIVC with passionate investigation efforts [19-22]. One explanation for this restricted achievement was due to the poor in vivo predictivity of the traditional in vitro dissolution methods. For example, the basket and paddle methods, two of the universally recommended dissolution systems, cannot offer gastrointestinal tract hydrodynamics mechanism, which is an important characteristic for the in vivo circumstances. The flow-through method, defined as the FDA official USP 4 apparatus, can provide a continuous flow of fresh dissolution medium, thus providing a perfect sink environment [23-25]. This dissolution approach offers a testing condition much closer to that of the gastrointestinal tract, and it was suggested as a potential tool for establishing IVIVC [26].

To further mimic the in vivo conditions, we modified the flow-through apparatus with open-loop configuration by positioning the flow-through cell on a shaker, which simulates mechanical destructive forces due to intestinal motility. The paracetamol immediate release (IR) tablets and felodipine extended release (ER) tablets were selected as the model drugs, and they were classified as "class I" drug and "class II" drug, respectively, based on biopharmaceutics classification system [27-29]. In the present study, the in vitro dissolution characteristics between the generic and innovator products of the model drugs were investigated and compared using modified flow-through method and compendial method, respectively. In vitro dissolution data were correlated with in vivo absorption profiles obtained from healthy volunteers. A level A IVIVC analysis and profile comparisons were applied to assess the predictive performance of the modified flow-through method.

\section{Materials and Methods}

2.1. Materials. Standards of paracetamol and felodipine were purchased from National Institutes for Food and Drug Control (Beijing, China). Hydrochloric acid (analytical grade) was obtained from Sinopharm Chemical Reagent Co., Ltd., and polysorbate 80 (analytical grade) was purchased from Tianjin Fengchuan Chemical Reagent Science and Technology Co., Ltd.
2.2. Formulations. Five different products of $500 \mathrm{mg}$ paracetamol IR tablets obtained from commercial sources within their shelf life period were studied: Ouhuatai ${ }^{\circledR}$ (test 1), Guangzhou Ouhua Pharmaceutical Co., Ltd., China; Jinshi ${ }^{\circledR}$ (test 2), Jinshi General Pharmaceutical Factory, China; Haoyisheng ${ }^{\circledR}$ (test 3), Sichuan Good Doctor-Panxi Pharmaceutical Co, Ltd., China; Nanjixue ${ }^{\circledR}$ (test 4), Guangdong Medi-world Pharmaceutical Co., Ltd., China; Panadol ${ }^{\circledR}$, Sino-American Tianjin SmithKline and French Lab., Ltd., China, which was used as the reference product (innovator). Four different products of $5 \mathrm{mg}$ felodipine ER tablets obtained/provided from commercial sources within their shelf life period were studied: HINYE $^{\circledR}$ (test 1 ), provided by Hunan HINYE pharmaceuticals Co. Ltd., China; Lifeon ${ }^{\circledR}$ (test 2), Hefei Lifeon Pharmaceutical Co. Ltd., China; Yitexin ${ }^{\circledR}$ (test 3), Nanjing EASEHEAL Pharmaceutical Co. Ltd., China; Plendil ${ }^{\circledR}$, AstraZeneca Pharmaceutical Co., Ltd., which was used as the reference product (innovator).

\subsection{Quantitative Analysis of Paracetamol and Felodipine.}

The amounts of paracetamol dissolved in the samples were analyzed by a microvolume UV-vis spectrophotometer (Quawell, San Jose, CA, USA) using $1 \mathrm{~mm}$ path absorbance for spectroscopic measurements, and the samples were analyzed at $\lambda=244 \mathrm{~nm}$. The dissolved felodipine levels in the samples were analyzed using a validated reversed phase HPLC system. An Agilent TC-C18 column $(4.6 \mathrm{~mm} \times 150 \mathrm{~mm}, 5 \mu \mathrm{m}$, Walbdbrom, Germany) was employed, and the mobile phase consisted of water: methanol $(10: 90, v / v)$. The flow rate was $1.0 \mathrm{~mL} / \mathrm{min}$, and the injection volume was $20 \mu \mathrm{L}$. The detection wavelength was set at $362 \mathrm{~nm}$.

Paracetamol concentrations in plasma were determined by HPLC with UV detection. The mobile phase was acetonitrile-water in a ratio of 20:80 $(v / v)$. The flow rate was $1.0 \mathrm{~mL} / \mathrm{min}$, and the column temperature was $30^{\circ} \mathrm{C}$. The injection volume was $20 \mu \mathrm{L}$, and chromatograms were recorded at $244 \mathrm{~nm}$. The levels of felodipine in the plasma samples were quantified using an Agilent 6460 triple quadrupole LC-MS/MS system (Agilent, Waldbronn, Germany) equipped with an electrospray ionization source (ESI). The mobile phase consisted of acetonitrile- $7 \mathrm{mM}$ ammonium acetate solution (including $0.05 \%$ formic acid) in a ratio of $63: 37(v / v)$ at a flow rate of $0.28 \mathrm{~mL} / \mathrm{min}$. The injection volume was $10 \mu \mathrm{L}$, and the column temperature was maintained at $40^{\circ} \mathrm{C}$. The mass spectrometer was operated in positive ion mode using multiple reactions monitoring (MRM). The precursor-product ion transition monitored was $\mathrm{m} / \mathrm{z}$ $384.1 \rightarrow 338.0$. The other optimized MS/MS parameters were as follows: fragmentor voltage $150 \mathrm{~V}$, collision energy $2 \mathrm{eV}$, source temperature $350^{\circ} \mathrm{C}$, drying gas $\left(\mathrm{N}_{2}\right)$ flow $4 \mathrm{~L} / \mathrm{min}$, nebulizer pressure $137.9 \mathrm{kPa}$, and capillary voltage $4 \mathrm{kV}$.

\subsection{Dissolution Testing}

2.4.1. Quality Control (QC) Method. The compendial QC method (Chinese Pharmacopoeia) using the basket for paracetamol IR tablet consisted of $900 \mathrm{~mL}$ hydrochloric acid water solution $(\mathrm{pH} 1.2)$ as a dissolution medium at $37 \pm 0.5^{\circ} \mathrm{C}$, and the basket rotation speed was $100 \mathrm{rpm}$. 


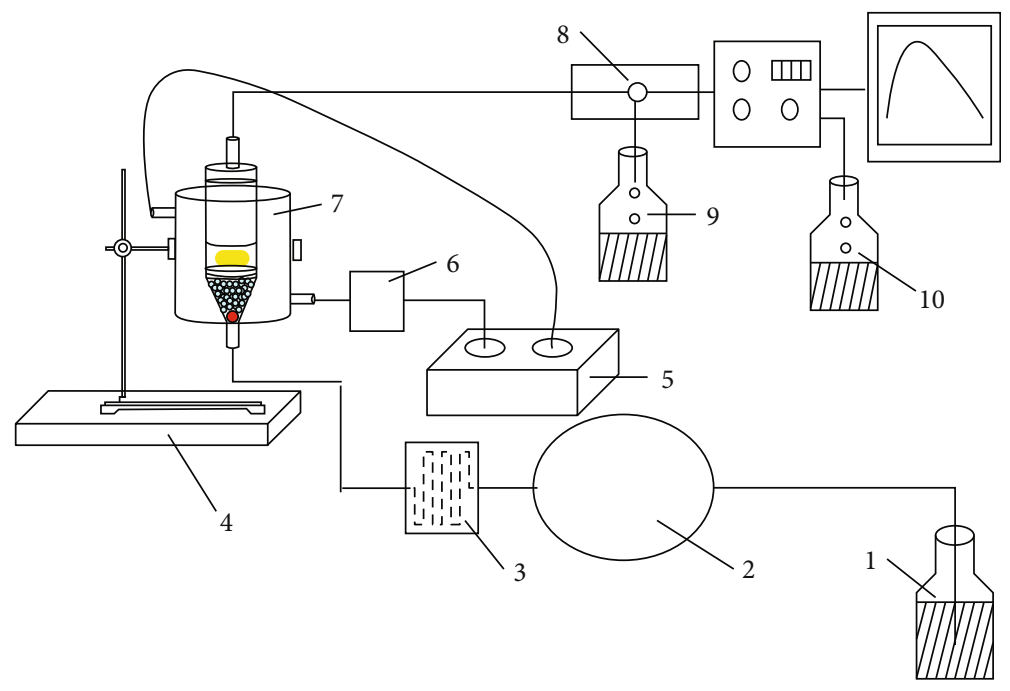

Figure 1: Schematic diagram of the flow-through dissolution apparatus. 1: dissolution media bottle; 2: high-precision liquid inlet pump; 3: heat exchange heater; 4: shaker; 5: thermostatic water bath; 6: peristaltic pump; 7: flow-through cell; 8: three-way valve; 9: waste collection bottle; 10: waste collection bottle.

Experiments were conducted in twelve replicates. Sample aliquots were withdrawn at $5,10,15,20,25,30,45$, and $60 \mathrm{~min}$ and replaced with an equal volume of fresh medium to maintain a constant total volume. The samples were filtered through a $0.45 \mu \mathrm{m}$ filter and then analyzed spectrophotometrically at $244 \mathrm{~nm}$.

The compendial QC method (USP 35) using the basket for felodipine ER tablet consisted of $500 \mathrm{~mL} 1 \%(w / v)$ polysorbate 80 as a dissolution medium at $37 \pm 0.5^{\circ} \mathrm{C}$, and the basket rotation speed was $100 \mathrm{rpm}$. Experiments were conducted in twelve replicates. Sample aliquots were withdrawn at $1,2,3,4,5,6,7$, and $8 \mathrm{~h}$ and replaced with an equal volume of fresh medium to maintain a constant total volume. The samples were filtered through a $0.45 \mu \mathrm{m}$ filter and then analyzed by HPLC.

2.4.2. Modified Flow-Through Method. The modified flowthrough apparatus is based on the principle of the USP apparatus 4. As presented in Figure 1, the modified assembly consists of a reservoir, a pump for the dissolution medium, a flow-through cell (diameters: $22.6 \mathrm{~mm}$ ) positioned on a shaker, and a water bath. One glass bead of $5 \mathrm{~mm}$ diameter was positioned in the apex of the flow-through cell, the lower conical part of the cell was filled with glass beads of $1-\mathrm{mm}$ diameter, and a glass microfiber filter (GF/D Whatman) was placed on the top of the cell. The temperature of the flow cell unit was maintained at $37.0 \pm 0.5^{\circ} \mathrm{C}$.

Experiments for paracetamol IR tablets and felodipine ER tablets were conducted in twelve replicates in an open system. The dissolution media were warmed up to $37.0 \pm 0.5^{\circ} \mathrm{C}$. The dissolution medium, prepared according to compendial method, was $\mathrm{pH} 1.2$ hydrochloric acid water solution for paracetamol and $1 \%(w / v)$ polysorbate 80 for felodipine. The agitational speed of the shaker was $100 \mathrm{rpm}$, and the optimized flow rate of the media was set as 14 and $4 \mathrm{~mL} / \mathrm{min}$ for paracetamol IR tablets and felodipine ER tablets, respectively. For each interval, samples were collected in clean tubes at the fol- lowing times: $2,4,6,8,10,15,20,25,30,35,40,45,50,55$, and $60 \mathrm{~min}$ for paracetamol and every $0.5 \mathrm{~h}$ up to $8 \mathrm{~h}$ for felodipine.

2.5. Comparative Bioavailability Studies. Paracetamol IR tablets bioavailability study: this study was an open, single-dose, randomized study with a cross-over design carried out in accordance with the guidelines of the Declaration of Helsinki. Twelve healthy male volunteers participated in the study (aged 19-26 years, weight $52-70 \mathrm{~kg}$ ). Each subject was assigned randomly and administered each of the three products (dose: $500 \mathrm{mg}$; products: test 1 , test 3 , and reference) according to a complete crossover Latin square design $(3 \times 3)$ with a washout period of 3 days. Blood samples $(3 \mathrm{~mL})$ were collected at the following time points: 0 (predose), 10, 20, 30, 40, 50, $60 \mathrm{~min}, 1,1.25,1.5,2,3,4,6,8$, and $10 \mathrm{~h}$ after drug intake.

Felodipine ER tablets bioavailability study: in an openlabeled, randomized, two-way crossover design, 20 male healthy volunteers (aged 22-30 years, weight $55-76 \mathrm{~kg}$ ) were randomly divided into two groups to receive the felodipine reference tablet followed by the test 1 tablet (dose: $10 \mathrm{mg}$ ), or vice versa, with 7-day washout period between doses. The venous blood samples were collected at $0.5,1.0,1.5$, $2.0,3.0,4.0,5.0,6.0,8.0,10.0,12.0,16.0,24.0,36.0$, and $48.0 \mathrm{~h}$ after dosing.

2.6. Modeling of Drug Release/Absorption Kinetics. To characterize paracetamol IR tablet and felodipine ER tablet release/absorption kinetics, a model-dependent approach using zero-order, first-order, and Weibull mathematical equations was applied. The mathematical model that best expressed the dissolution/absorption profile of paracetamol IR tablets and felodipine ER tablets was selected based on the coefficient of determination $\left(R^{2}\right)$ and model selection criteria (MSC). The modeling was performed using the DDSolver software (a menu-driven add-in program in Microsoft Excel) $[30,31]$. 


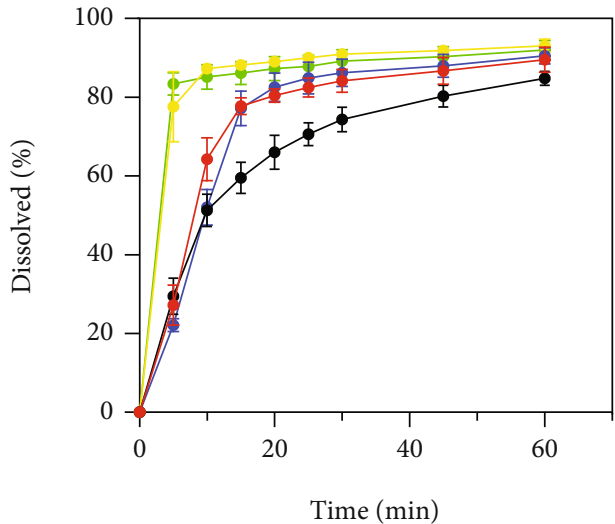

(a)

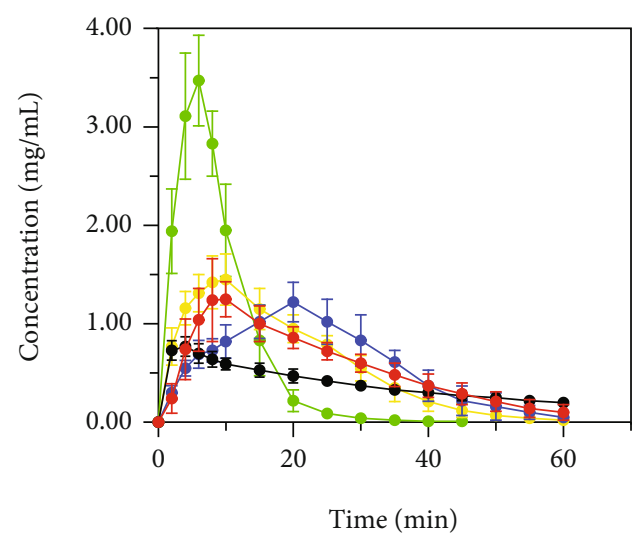

(b)

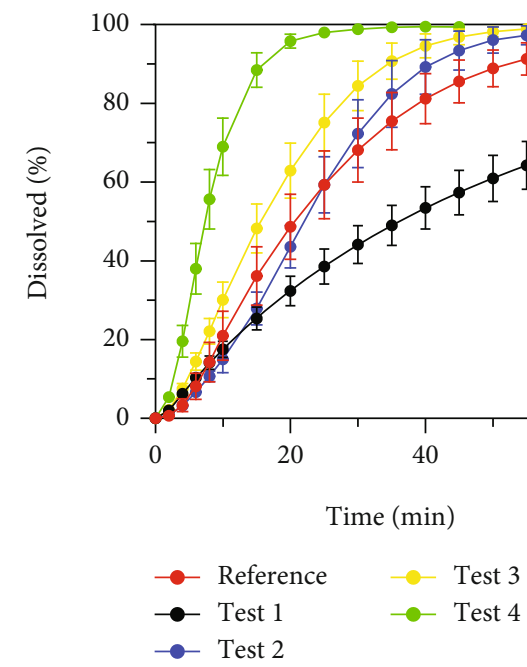

(c)

FIGURE 2: Dissolution profile comparison of paracetamol IR tablets using QC method and modified flow-through method. (a) The cumulative dissolution profiles using QC method (basket); (b) the differential dissolution profiles using modified flow-through method; (c) the cumulative dissolution profiles integrated from differential dissolution profiles. All data are the mean $\pm \mathrm{SD}, n=12$.

2.7. Similarity of In Vitro Dissolution Profiles. In vitro dissolution profile sameness was assessed using the modelindependent approach-the similarity factor $\left(f_{2}\right.$, Equation (1)) $[10,11]$.

$$
f_{2}=50 \cdot \log \left\{\left[1+\frac{1}{n} \sum_{t=1}^{n}\left(R_{t}-T_{t}\right)^{2}\right]^{-0.5} \times 100\right\},
$$

where $n$ is the number of time points and $R_{t}$ and $T_{t}$ are the dissolution value of the reference and test formulations at time $t$, respectively. The $f_{2}$ is basically a measurement of the similarity in the percent drug dissolved between the two curves. An $f_{2}$ value greater than 50 (50-100) demonstrates similarity of the curves.

2.8. In Vitro-In Vivo Correlations. The average plasma concentration versus time curve of paracetamol IR tablets/felodipine ER tablets was transformed into the percent dose absorbed versus time using Wagner-Nelson or LooRiegelman method based on their determined compartmen- tal models [32-35]. The Wagner-Nelson method is suitable for one-compartment model, and the Loo-Riegelman method fits for the two-compartment model.

Level A IVIVCs for paracetamol IR tablets/felodipine ER tablets were evaluated by plotting the mean percent drug dissolved $\left(F_{\text {dis }}\right)$ from modified flow-through method/QC method versus the mean percent drug absorbed $\left(F_{\text {abs }}\right)$, respectively. Linear regression analysis was used to evaluate the correlations. The $F_{\text {abs }}$ versus $F_{\text {dis }}$ curve comparisons of paracetamol IR tablets and felodipine ER tablets were also examined using the estimated release/absorption kinetics parameters.

\section{Results and Discussion}

\subsection{Dissolution Testing}

3.1.1. QC Method. Figure 2(a) and Figure 3(a) show the dissolution profiles of paracetamol IR tablets and felodipine ER tablets using the QC method, respectively. For paracetamol IR tablets, the test 2 product showed dissolution 


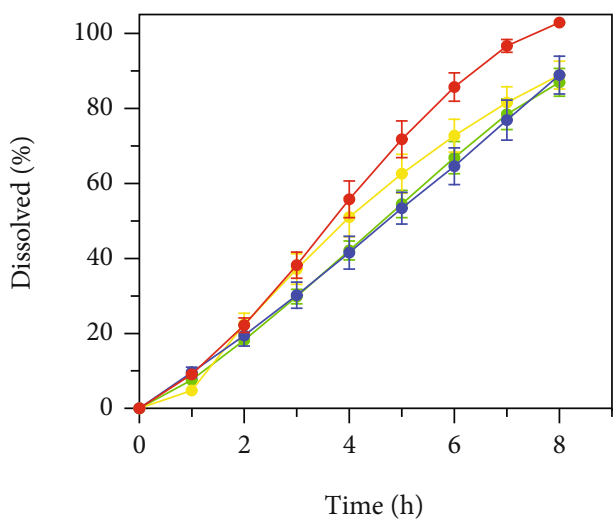

(a)

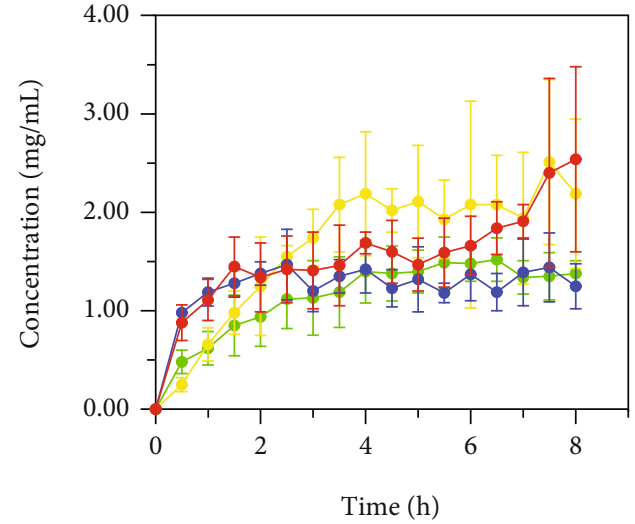

(b)

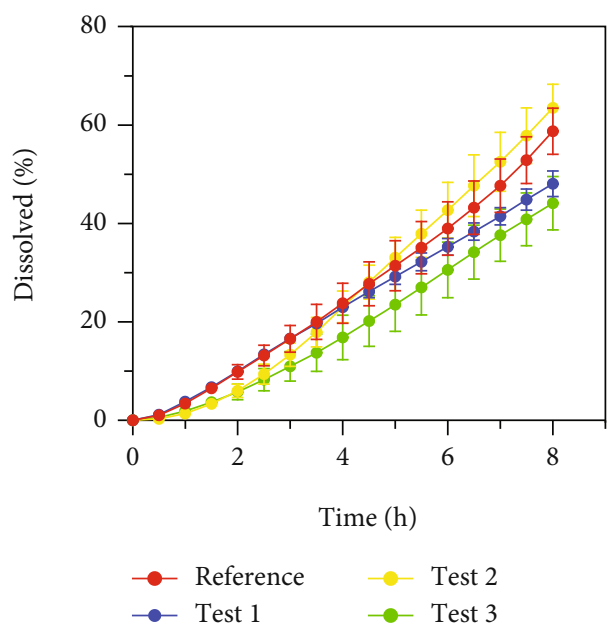

(c)

FIGURE 3: Dissolution profiles comparison of felodipine ER tablets using QC method and modified flow-through method. (a) The cumulative dissolution profiles using QC method (basket); (b) the differential dissolution profiles using modified flow-through method; (c) the cumulative dissolution profiles integrated from differential dissolution profiles. All data are the mean $\pm \mathrm{SD}, n=12$.

similarity with the reference product $\left(f_{2}=65.06\right)$, while there were no similarities for test 1 , test 3 , and test 4 products compared with the reference formulation $\left(f_{2}=47.33,34.29\right.$, and 33.11 , respectively). Test 3 and test 4 products dissolved fastest (approximately $85 \%$ of the labeled drug dissolved in $10 \mathrm{~min}$ ), the reference and test 2 products dissolved about $85 \%$ in $30 \mathrm{~min}$, and test 1 dissolved slowest (only 75\% of the labeled drug dissolved in $30 \mathrm{~min}$ ). More than $85 \%$ drug released was observed in all products within $60 \mathrm{~min}$. For felodipine ER tablets, test 2 product had $f_{2}$ similarity with the reference product $\left(f_{2}=53.05\right)$, while there are no similarities for test 1 and test 3 products compared with the reference $\left(f_{2}=42.66\right.$ and 43.61 , respectively). More than $85 \%$ drug was released in $8 \mathrm{~h}$ for all felodipine products.

3.1.2. Modified Flow-Through Method. The differential dissolution profiles of paracetamol IR tablets and felodipine ER tablets using the modified flow-through method are shown in Figure 2(b) and Figure 3(b), respectively. The corresponding cumulative dissolution profiles were integrated from differential dissolution profiles, which are shown in Figure 2(c) and Figure 3(c), respectively. As demonstrated in Figure 2(b), the differential dissolution profiles of paracetamol test 1 and test 4 products exhibited significant differences compared with that of reference product, and test 3 product had similar profile with the reference. For example, the peak concentration $\left(C_{\max }\right.$ dissolution $)$ of test 1 is much lower than the reference, while the $C_{\max }$ dissolution of test 4 is approximately 2.8 fold higher than the reference. These differences in generic and reference products resulted in corresponding discrepancies in cumulative dissolution profiles (shown in Figure 2(c)). The $f_{2}$ value (62.80) obtained from test 2 and reference comparison was similar with that of QC method (65.06), while the $f_{2}$ values for test 1 , test 3 , and test $4(36.58,47.05$, and 20.96 , respectively) showed large differences compared with the QC method (47.33, 34.29, and 33.11, respectively). The dissolution profiles obtained from modified flow-through method are more smoothly and discriminative compared with the QC basket method, which might be suitable to detect small product deviations. For felodipine ER tablets, although the differential dissolution profiles of generic products exhibited significant differences compared with that of reference 
TABLE 1: Mathematical models and release/absorption kinetics parameters comparisons for paracetamol IR tablets and felodipine ER tablets.

\begin{tabular}{|c|c|c|c|c|c|c|}
\hline \multicolumn{2}{|l|}{ Formulations } & $\begin{array}{l}\text { Dissolution/deconvolution } \\
\text { methods }\end{array}$ & Dissolution models & Model parameters & $R^{2}$ & MSC \\
\hline \multicolumn{7}{|c|}{ Paracetamol IR tablets } \\
\hline \multirow{2}{*}{\multicolumn{2}{|c|}{ Reference }} & QC method & \multirow{6}{*}{ Weibull } & $\mathrm{Td}=12.164 \quad \beta=0.802$ & 0.8678 & 1.52 \\
\hline & & Modified flow-through method & & $\mathrm{Td}=27.661 \quad \beta=1.412$ & 0.9976 & 5.79 \\
\hline \multirow{9}{*}{ In vitro dissolved } & \multirow{2}{*}{ Test 1} & QC method & & $\mathrm{Td}=19.019 \quad \beta=0.639$ & 0.9756 & 3.21 \\
\hline & & Modified flow-through method & & $\mathrm{Td}=52.734 \quad \beta=0.991$ & 0.999 & 6.61 \\
\hline & \multirow{2}{*}{ Test 2} & QC method & & $\mathrm{Td}=13.241 \quad \beta=1.090$ & 0.9129 & 1.94 \\
\hline & & Modified flow-through method & & $\mathrm{Td}=26.470 \quad \beta=1.891$ & 0.9997 & 7.85 \\
\hline & \multirow{2}{*}{ Test 3} & QC method & \multirow{5}{*}{$F=100 *\left\{1-\operatorname{Exp}\left[-\left((t / \mathrm{Td})^{\wedge} \beta\right)\right]\right\}$} & $\mathrm{Td}=0.656 \quad \beta=0.230$ & 0.9083 & 1.89 \\
\hline & & Modified flow-through method & & $\mathrm{Td}=19.956 \quad \beta=1.524$ & 0.9999 & 8.62 \\
\hline & \multirow{2}{*}{ Test 4} & QC method & & $\mathrm{Td}=0.064 \quad \beta=0.129$ & 0.9579 & 2.67 \\
\hline & & Modified flow-through method & & $\mathrm{Td}=9.255 \quad \beta=1.717$ & 0.9986 & 6.24 \\
\hline & Reference & Wagner-Nelson method & & $\mathrm{Td}=22.254 \quad \beta=2.303$ & 0.9986 & 5.75 \\
\hline \multirow[t]{2}{*}{ In vivo absorbed } & Test 1 & Wagner-Nelson method & & $\mathrm{Td}=20.629 \quad \beta=1.588$ & 0.9959 & 4.69 \\
\hline & Test 2 & Wagner-Nelson method & & $\mathrm{Td}=19.318 \quad \beta=1.297$ & 0.9374 & 1.97 \\
\hline \multicolumn{7}{|c|}{ Felodipine ER tablets } \\
\hline \multirow{2}{*}{\multicolumn{2}{|c|}{ Reference }} & QC method & & $k 0=13.547$ & 0.9857 & 3.99 \\
\hline & & Modified flow-through method & & $k 0=6.608$ & 0.9729 & 3.48 \\
\hline \multirow{2}{*}{\multicolumn{2}{|c|}{ Test 1}} & QC method & Zero-order & $k 0=10.829$ & 0.996 & 5.28 \\
\hline & & Modified flow-through method & & $k 0=5.860$ & 0.9939 & 4.98 \\
\hline \multirow{4}{*}{ In vitro dissolved } & \multirow{2}{*}{ Test 2} & QC method & & $k 0=11.747$ & 0.9809 & 3.71 \\
\hline & & Modified flow-through method & & $k 0=6.987$ & 0.9307 & 2.54 \\
\hline & \multirow{2}{*}{ Test 3} & QC method & & $k 0=10.887$ & 0.9929 & 4.69 \\
\hline & & Modified flow-through method & $F=k 0 * t$ & $k 0=5.011$ & 0.9526 & 2.92 \\
\hline \multirow{2}{*}{ In vivo absorbed } & Reference & Loo-Riegelman method & & $k 0=7.046$ & 0.9814 & 3.7 \\
\hline & Test 1 & Loo-Riegelman method & & $k 0=7.765$ & 0.9914 & 4.48 \\
\hline
\end{tabular}

$F$ is the fraction (\%) of drug released at time $t$; $\mathrm{Td}$ is the mean dissolution time, when $63.2 \%$ of the drug in the dosage form has been released; $\beta$ is the shape parameter; $k 0$ is the zero-order release constant.

product, their corresponding cumulative dissolution profiles were similar (shown in Figure 3(c)), with the $f_{2}$ values 68.64, 73.10 , and 55.30 for test 1 , test 2 , and test 3 compared with reference, respectively. These $f_{2}$ values showed a large difference compared with the corresponding values $(42.66,53.05$, and 43.61, respectively) obtained from QC method. The total amounts of drug released were much lower than that of QC method; only no more than $60 \%$ drug released was observed in all products within $8 \mathrm{~h}$.

3.2. Evaluation of Release/Absorption Kinetics. The model parameters, determination coefficient $\left(R^{2}\right)$, and MSC were determined for all formulations and presented in Table 1. According to the $R^{2}$ and MSC, the dissolution/absorption profiles of paracetamol IR tablets were best described by the Weibull model, and acceptable fitting results were also achieved by first-order model. For felodipine ER tablets, the in vitro drug release/in vivo absorption profiles were best explained by the zero-order model.

\subsection{IVIVC Analysis and Profile Comparisons}

3.3.1. Deconvolution of In Vivo Data. According to the minimum Akaike's information criterion (AIC) estimation of the compartment models using Phoenix WinNonlin 6.1 software, the concentration-time profile of paracetamol IR tablets was best described by one-compartment model, and the in vivo profile characteristics of felodipine ER tablets was best described by two-compartment model. Therefore, the Wagner-Nelson method was employed for deconvolution of paracetamol IR tablets in vivo data, and the LooRiegelman method was applied to felodipine ER tablets. Figures $4(\mathrm{a})-4(\mathrm{c})$ show the $F_{\text {abs }}$ profiles of paracetamol reference, test 1 , and test 3 products, respectively, obtained by the Wagner-Nelson method, and Figures 5(a) and 5(b) present the $F_{\text {abs }}$ curves of felodipine reference and test 1 formulations, respectively, based on the Loo-Riegelman method.

3.3.2. Profile Comparisons. As demonstrated in Figures 4 and 5 , the $F_{\text {dis }}$ profiles of both paracetamol IR tablets and 


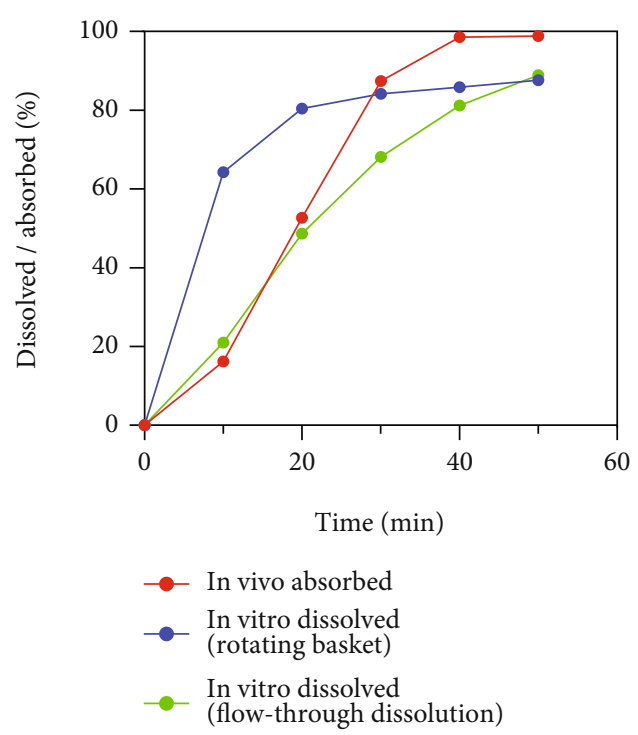

(a)

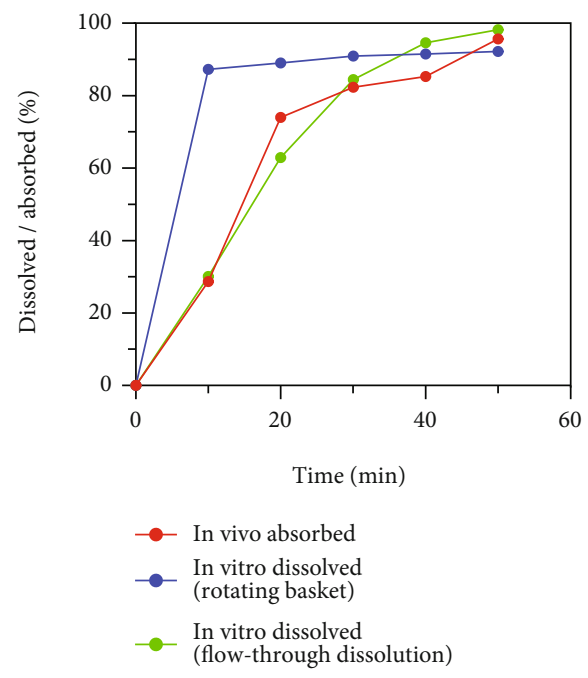

(c)

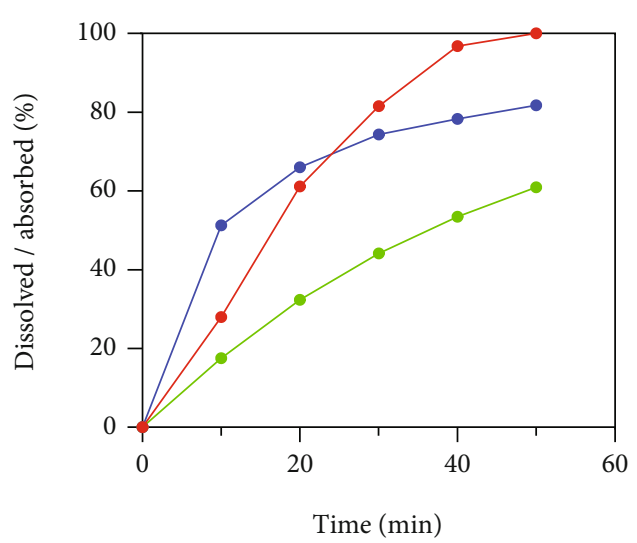

- - In vivo absorbed

- In vitro dissolved (rotating basket)

_- In vitro dissolved (flow-through dissolution)

(b)

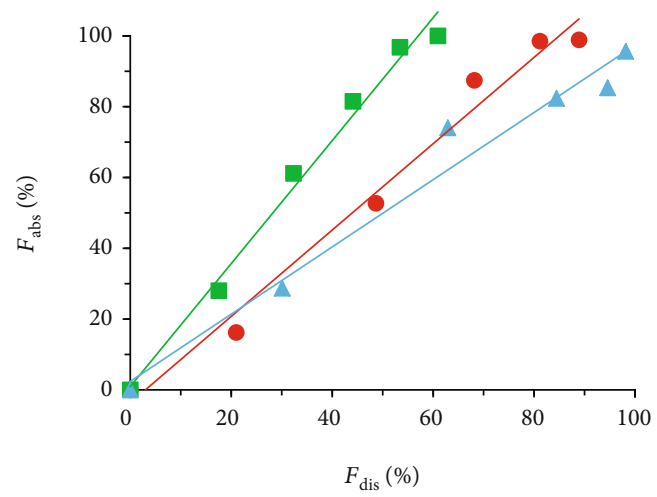

- Reference

- Test 1

A Test 3

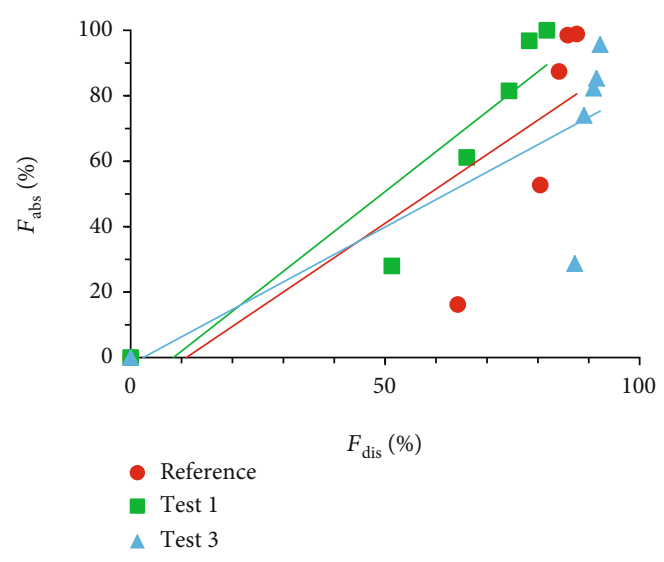

(e)

FIGURE 4: Profile comparison and IVIVC analysis of the in vitro percent drug dissolved $\left(F_{\text {dis }}\right)$ versus the in vivo percent drug absorbed $\left(F_{\text {abs }}\right)$ of paracetamol IR tablets. (a) In vitro dissolved and in vivo absorbed profiles for paracetamol reference tablet; (b) in vitro dissolved and in vivo absorbed profiles for paracetamol test 1 tablet; (c) in vitro dissolved and in vivo absorbed profiles for paracetamol test 3 tablet; linear regression plots of mean $F_{\text {abs }}$ versus mean $F_{\text {dis }}$ obtained from modified flow-through method (d) and QC method (e). All in vitro $(n=12)$ and in vivo $(n=4)$ data are the mean $\pm \mathrm{SD}$. 

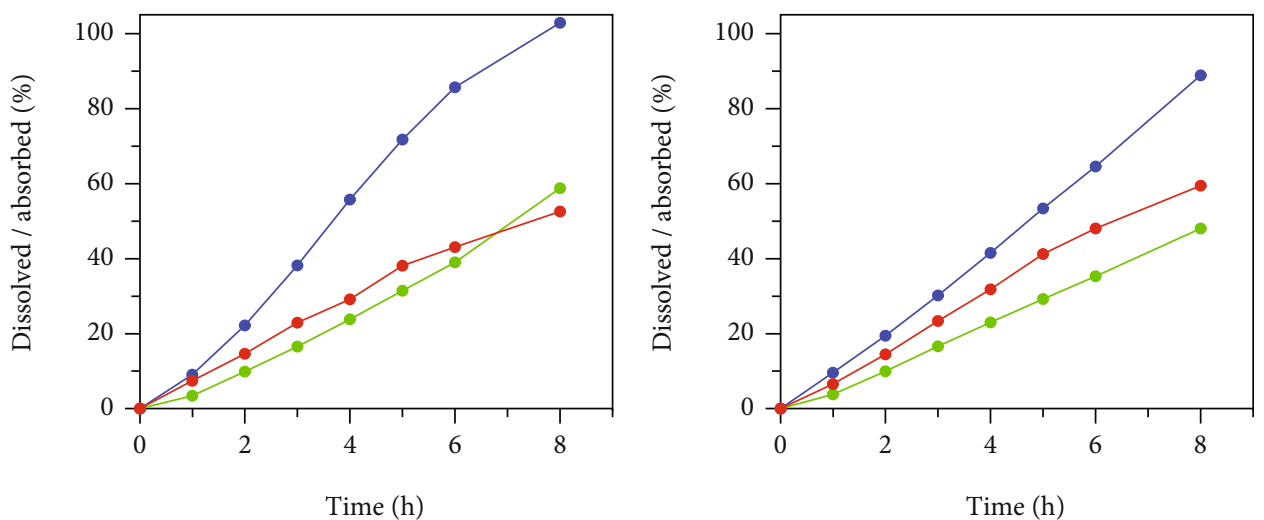

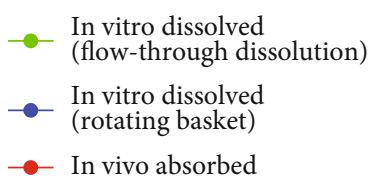

(a)

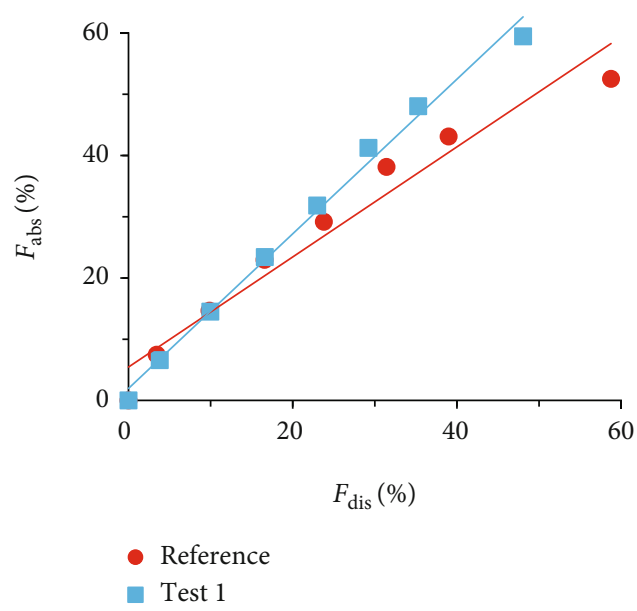

(c)

$$
\begin{aligned}
& - \text { In vitro dissolved } \\
& \text { (flow-through dissolution) } \\
& -\quad \begin{array}{l}
\text { In vitro dissolved } \\
\text { (rotating basket) }
\end{array} \\
& -\quad \text { In vivo absorbed }
\end{aligned}
$$

(b)

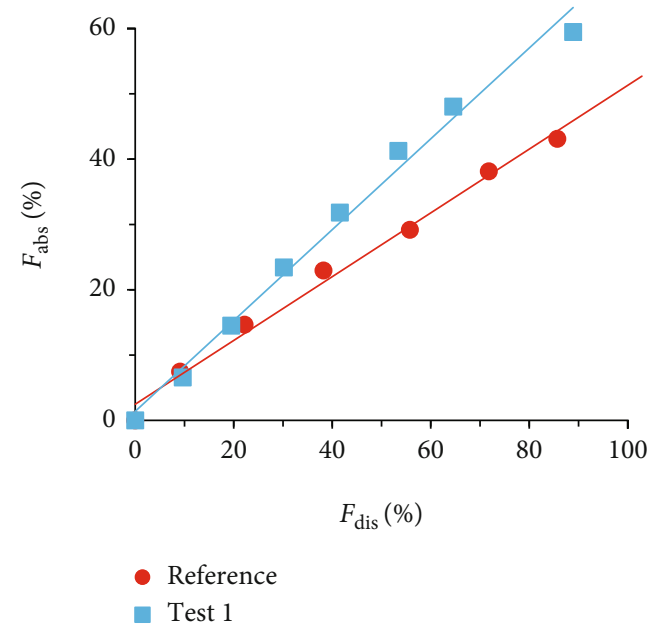

(d)

Figure 5: Profiles comparison and IVIVC analysis of the in vitro percent drug dissolved $\left(F_{\text {dis }}\right)$ versus the in vivo percent drug absorbed $\left(F_{\text {abs }}\right)$ of felodipine ER tablets. (a) In vitro dissolved and in vivo absorbed profiles for felodipine reference tablet; (b) in vitro dissolved and in vivo absorbed profiles for felodipine test 1 tablet; linear regression plots of mean $F_{\text {abs }}$ versus mean $F_{\text {dis }}$ obtained from modified flow-through method $(\mathrm{c})$ and QC method $(\mathrm{d})$. All in vitro $(n=12)$ and in vivo $(n=10)$ data are the mean \pm SD.

felodipine ER tablets from the QC methods were steeper than the corresponding in vivo $F_{\text {abs }}$ profiles, while the $F_{\text {dis }}$ profiles obtained from modified flow-through method were similar or slightly lower than the $F_{\text {abs }}$ profiles. Figures 4(a)-4(c) show that the dissolution profiles of paracetamol IR tablets obtained from the QC method displayed a high dissolution at initial times and a lower dissolution rate after $30 \mathrm{~min}$ than the in vivo absorption profiles. In contrast, smooth dissolution profiles were obtained by the modified flow-through method. The dissolution profile of paracetamol reference product obtained by the modified flow-through method was similar with the absorption profile at initial time (20 min), afterward the dissolution rate was lower than the absorption rate. The dissolution profile of paracetamol test 1 was completely lower than the absorption profile during the whole period of time, while in vitro profile of paracetamol test 3 nearly overlapped with the in vivo absorption profile. Figures 5(a) and 5(b) show that the dissolution rate of felodipine reference and test 1 products using QC method was significantly larger than the absorption rate, while the in vitro rate was comparable with in vivo rate in terms of modified flow-through method.

3.3.3. In Vitro-In Vivo Correlation. Figures 4(d) and 4(e) and Figures 5(c) and 5(d) demonstrate the level A IVIVC plots of paracetamol IR tablets and felodipine ER tablets, respectively. For paracetamol IR tablets, the QC method did not show correlations, while linear regression analysis of $F_{\text {abs }}$ versus $F_{\text {dis }}$ resulted in a significant correlation $\left(R^{2}>0.98\right)$ for the three formulations in terms of the modified flow-through method. The in vitro dissolution of reference and test 3 from modified flow-through method were close to the in vivo absorption, as 
indicated by the estimated regression slopes (1.2199 and 0.9450 for reference and test 3 , respectively), and the in vitro dissolution of test 1 ran behind of its in vivo absorption (the regression slope $=1.7359$ ). For felodipine ER tablets, both the QC and modified flow-through methods yield essentially linear correlations $\left(R^{2}>0.96\right)$. The in vitro release of felodipine reference and test 1 ran ahead of in vivo absorption using QC method (regression slopes of 0.4883 and 0.6953 for felodipine reference and test 1 ), and the in vitro release using modified flow-through method was close to the in vivo absorption (regression slopes of 0.8990 and 1.2526 for felodipine reference and test 1). The IVIVC analysis indicate that the modified flow-through method could reasonably reflect the in vivo absorption of paracetamol IR tablets and felodipine ER tablets.

\section{Conclusion}

The modified flow-through method can provide the differential dissolution profiles of the formulations and is able to discriminate the subtle instantaneous release differences between the generic products and innovator. The discriminatory power of modified flow-through method is superior to the QC compendial method and achieve good correlations with the in vivo absorption data. Our study demonstrated that the modified flow-through method is a potential powerful tool for predicting the in vivo performance of formulations.

\section{Data Availability}

The data used to support the findings of this study are included within the article.

\section{Conflicts of Interest}

The authors declare that there is no conflict of interest regarding the publication of this article.

\section{Acknowledgments}

This work was supported by the National Natural Science Foundation of China (project number: 82073940).

\section{References}

[1] G. Zhang, M. Sun, S. Jiang et al., "Investigating a modified apparatus to discriminate the dissolution capacity _in vitro_ and establish an IVIVC of mycophenolate mofetil tablets in the fed state," Journal of Pharmaceutical Sciences, vol. 110, no. 3, pp. 1240-1247, 2021.

[2] G. F. Gao, M. Ashtikar, R. Kojima et al., "Predicting drug release and degradation kinetics of long-acting microsphere formulations of tacrolimus for subcutaneous injection," Journal of Controlled Release, vol. 329, pp. 372-384, 2021.

[3] E. Sjögren, B. Abrahamsson, P. Augustijns et al., “_In vivo_ methods for drug absorption - Comparative physiologies, model selection, correlations with _ in vitro_ methods (IVIVC), and applications for formulation/API/excipient characterization including food effects," European Journal of Pharmaceutical Sciences, vol. 57, pp. 99-151, 2014.
[4] V. H. Sunesen, B. L. Pedersen, H. G. Kristensen, and A. Müllertz, "In vivo in vitro correlations for a poorly soluble drug, danazol, using the flow-through dissolution method with biorelevant dissolution media," European Journal of Pharmaceutical Sciences, vol. 24, no. 4, pp. 305-313, 2005.

[5] V. R. S. Uppoor, "Regulatory perspectives on in vitro (dissolution)/in vivo (bioavailability) correlations," Journal of Controlled Release, vol. 72, no. 1-3, pp. 127-132, 2001.

[6] B. Sánchez-Dengra, I. González-García, M. González-Álvarez, I. González-Álvarez, and M. Bermejo, "Two-step _in vitro__in vivo_ correlations: deconvolution and convolution methods, which one gives the best predictability? Comparison with one-step approach," European Journal of Pharmaceutics and Biopharmaceutics, vol. 158, pp. 185-197, 2021.

[7] P. Jain, R. S. Pawar, R. S. Pandey et al., "In-vitro in-vivo correlation (IVIVC) in nanomedicine: is protein corona the missing link?," Biotechnology Advances, vol. 35, no. 7, pp. 889-904, 2017.

[8] J. V. Andhariya, J. Shen, S. Choi, Y. Wang, Y. Zou, and D. J. Burgess, "Development of _in vitro-in vivo_ correlation of parenteral naltrexone loaded polymeric microspheres," Journal of Controlled Release, vol. 255, pp. 27-35, 2017.

[9] M. A. Nguyen, T. Flanagan, M. Brewster et al., "A survey on IVIVC/IVIVR development in the pharmaceutical industry past experience and current perspectives," European Journal of Pharmaceutical Sciences, vol. 102, pp. 1-13, 2017.

[10] FDA CDER, Guidance for industry: dissolution testing of immediate-release solid oral dosage forms, Food and Drug Administration, Center for Drug Evaluation and Research (CDER), 1997.

[11] FDA, Guidance for industry: extended release oral dosage forms: development, evaluation, and application of in vitro/in vivo correlations, Food and Drug Administration, Rockville, MD, 1997.

[12] R. C. Rossi, C. L. Dias, L. Bajerski, A. M. Bergold, and P. E. Fröehlich, "Development and validation of discriminating method of dissolution for fosamprenavir tablets based on in vivo data," Journal of Pharmaceutical and Biomedical Analysis, vol. 54, no. 3, pp. 439-444, 2011.

[13] M. G. Davanço, D. R. Campos, and P. D. O. Carvalho, “_In vitro_ - _in vivo_correlation in the development of oral drug formulation: a screenshot of the last two decades," International Journal of Pharmaceutics, vol. 580, article 119210, 2020.

[14] S. Suarez-Sharp, M. Li, J. Duan, H. Shah, and P. Seo, "Regulatory experience with in vivo in vitro correlations (IVIVC) in new drug applications," The AAPS Journal, vol. 18, no. 6, pp. 1379-1390, 2016.

[15] S. Gite, M. Chogale, and V. Patravale, "Development and validation of a discriminating dissolution method for atorvastatin delayed-release nanoparticles using a flow-through cell: a comparative study using USP apparatus 4 and 1," Dissolution Technologies, vol. 23, no. 2, pp. 14-20, 2016.

[16] Y. Saibi, H. Sato, and H. Tachiki, "Developing in vitro-in vivo correlation of risperidone immediate release tablet," AAPS PharmSciTech, vol. 13, no. 3, pp. 890-895, 2012.

[17] J. E. Tingstad and S. Riegelman, "Dissolution rate studies I: design and evaluation of a continuous flow apparatus," Journal of Pharmaceutical Sciences, vol. 59, no. 5, pp. 692-696, 1970.

[18] G. Levy, J. R. Leonards, and J. A. Procknal, "Interpretation of _in vitro_ dissolution data relative to the gastrointestinal absorption characteristics of drugs in tablets," Journal of Pharmaceutical Sciences, vol. 56, no. 10, pp. 1365-1367, 1967. 
[19] M. F. Mohamed, S. Trueman, A. A. Othman, J. Han, T. R. Ju, and P. Marroum, "Development of in vitro-in vivo correlation for upadacitinib extended-release tablet formulation," The AAPS Journal, vol. 21, no. 6, p. 108, 2019.

[20] A. al Durdunji, H. S. AlKhatib, and M. al-Ghazawi, "Development of a biphasic dissolution test for Deferasirox dispersible tablets and its application in establishing an in vitro-in vivo correlation," European Journal of Pharmaceutics and Biopharmaceutics, vol. 102, pp. 9-18, 2016.

[21] T. H. Kim, S. Shin, S. W. Jeong, J. B. Lee, and B. S. Shin, "Physiologically relevant in vitro-in vivo correlation (IVIVC) approach for sildenafil with site-dependent dissolution," Pharmaceutics., vol. 11, no. 6, p. 251, 2019.

[22] C. Mircioiu, V. Anuta, I. Mircioiu, A. Nicolescu, and N. Fotaki, "In vitro-in vivo correlations based on in vitro dissolution of parent drug diltiazem and pharmacokinetics of its metabolite," Pharmaceutics., vol. 11, no. 7, p. 344, 2019.

[23] M. Nicklasson, A. Orbe, J. Lindberg et al., "A collaborative study of the in vitro dissolution of phenacetin crystals comparing the flow through method with the USP paddle method," International Journal of Pharmaceutics, vol. 69, no. 3, pp. 255-264, 1991.

[24] M. Nicklasson, B. Wennergren, J. Lindberg et al., "A collaborative in vitro dissolution study using the flow-through method," International Journal of Pharmaceutics, vol. 37, no. 3, pp. 195202, 1987.

[25] B. Wennergren, J. Lindberg, M. Nicklasson et al., "A collaborative in vitro dissolution study: comparing the flow-through method with the USP paddle method using USP prednisone calibrator tablets," International Journal of Pharmaceutics, vol. 53, no. 1, pp. 35-41, 1989.

[26] S. Jiang, G. Zhang, L. Wang, Y. Zeng, W. Liu, and Z. Cheng, "Development of a two-compartment system in vitro dissolution test and correlation with in vivo pharmacokinetic studies for celecoxib," AAPS PharmSciTech, vol. 21, no. 2, 2020.

[27] R. Löbenberg and G. L. Amidonb, "Modern bioavailability, bioequivalence and biopharmaceutics classification system. New scientific approaches to international regulatory standards," European Journal of Pharmaceutics and Biopharmaceutics, vol. 50, no. 1, pp. 3-12, 2000.

[28] Z. Zhou, C. Dunn, I. Khadra, C. G. Wilson, and G. W. Halbert, "Influence of physiological gastrointestinal surfactant ratio on the equilibrium solubility of BCS class II drugs investigated using a four component mixture design," Molecular Pharmaceutics, vol. 14, no. 12, pp. 4132-4144, 2017.

[29] N. Katori, N. Aoyagi, and T. Terao, "Estimation of agitation intensity in the GI tract in humans and dogs based on in vitro/in vivo correlation," Pharmaceutical Research, vol. 12, no. 2, pp. 237-243, 1995.

[30] Y. Zhang, M. Huo, J. Zhou et al., "DDSolver: an add-in program for modeling and comparison of drug dissolution profiles," The AAPS Journal, vol. 12, no. 3, pp. 263-271, 2010.

[31] J. Zuo, Y. Gao, N. Bou-Chacra, and R. Löbenberg, "Evaluation of the DDSolver software applications," BioMed Research International, vol. 2014, 9 pages, 2014.

[32] X. Hu, J. Zhang, X. Tang et al., “An accelerated release method of risperidone loaded PLGA microspheres with good IVIVC," Current Drug Delivery, vol. 15, no. 1, pp. 87-96, 2018.

[33] R. Praveen, P. R. Prasad Verma, J. Venkatesan, D. H. Yoon, S. K. Kim, and S. K. Singh, “_In vitro_and _in vivo_evaluation of gastro-retentive carvedilol loaded chitosan beads using Gas- troplus ${ }^{\mathrm{TM}}$," International Journal of Biological Macromolecules, vol. 102, pp. 642-650, 2017.

[34] A. Simon, M. I. Amaro, A. M. Healy, L. M. Cabral, and V. P. de Sousa, "Comparative evaluation of rivastigmine permeation from a transdermal system in the Franz cell using synthetic membranes and pig ear skin with _in vivo-in vitro_correlation," International Journal of Pharmaceutics, vol. 512, no. 1, pp. 234-241, 2016.

[35] T. D. S. Honório, E. C. Pinto, H. V. A. Rocha et al., "In vitroin vivo correlation of efavirenz tablets using GastroPlus ${ }^{\circledR}$," AAPS PharmSciTech, vol. 14, no. 3, pp. 1244-1254, 2013. 